\title{
Ossified Metaplastic Spinal Meningioma Without Psammomatous Calcification: A Case Report
}

\author{
Taiki Murakami,* Shinji Tanishima," Chikako Takeda,* Shinsuke Kato $†$ and Hideki Nagashima* \\ *Division of Orthopedic Surgery, Department of Sensory and Motor Organs, School of Medicine, Tottori University Faculty of \\ Medicine, Yonago 683-8504, Japan, and †Division of Neuropathology, Department of Pathology, School of Medicine, Tottori University \\ Faculty of Medicine, Yonago 683-8504, Japan
}

\begin{abstract}
Meningiomas constitute approximately $25 \%$ of primary spinal cord tumors, and $1 \%$ to $5 \%$ are calcified. Ossification is a rare event and the etiology of ossification in meningiomas is not well known. We present the case of a 29-year-old female with a rare case of ossified thoracic spinal metaplastic meningioma. The tumor was successfully resected, and pathology confirmed ossified metaplastic meningioma. On histopathological examination, only mature bone tissue and tumor cells were present in the region containing no psammoma bodies, suggesting that the tumor cells had transitioned to mature osteocytes.
\end{abstract}

Key words calcification; metaplastic meningioma; ossified spinal cord tumor; thoracic spinal metaplastic meningioma

Spinal meningioma is the second most frequent spinal cord tumor following schwannoma, and it has been reported to account for $25-46 \%$ of all spinal cord tumor cases. ${ }^{1}$ Although calcification is common in cranial meningioma, gross calcification is rare in spinal tumors and is observed only in $1 \%$ to $5 \%$ of all spinal meningiomas. ${ }^{2,3}$ Ossification in spinal meningiomas is even more abnormal and is rarely reported. In the World Health Organization classification, the histological classification of ossified meningioma is a phenotype of metaplastic meningioma. Although the etiology of ossification in meningiomas is not well known, metaplasia of arachnoid cells/dystrophic calcification may be the cause. ${ }^{4}$ We report a patient with symptomatic ossified metaplastic spinal meningioma whose tumor was mostly composed of ossified tissue, and mature bone tissue was observed in the tumor tissue on histopathological examination.

Corresponding author: Taiki Murakami, MD

air-taiki@hotmail.co.jp

Received 2019 February 15

Accepted 2019 March 13

Online published 2019 June 20

Abbreviation: MEP, motor evoked potential
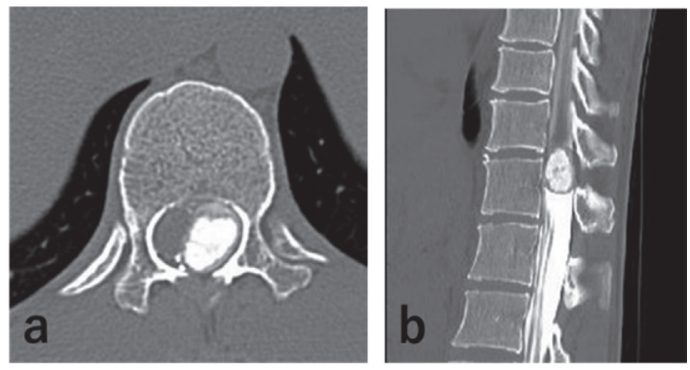

Fig. 1. Computed tomographic myelography showing a large totally ossified intradural round mass occupying more than half of the spinal canal at the T12 level. Axial view at T12 (a) and sagittal view (b).

\section{PATIENT REPORT}

A 29-year-old female developed low back pain without a trigger one year and 6 months prior to first visit, and pain and numbness appeared in the lateral region of the left lower limb after standing for a prolonged period one year prior to first visit, aggravating the low back pain. Abdominal pain developed 6 months prior to first visit, and the patient visited an obstetrics and gynecology clinic, but no abnormality was found. She visited an orthopedic clinic, and a spinal cord tumor was detected by thoracic spinal MRI, for which the patient was referred to our department. She was able to walk independently without any aid, and no hypoesthesia of perception or sense of vibration was noted. The patellar and Achilles tendon reflexes were normal on both sides, and the Babinski reflex was also negative on both sides. Dysuria and lower limb muscle weakness were absent. An ossified lesion was present at the T12 vertebral level on plain radiography of the lumbar spine. On myelo-CT, a high-density mass was present in the dura, completely occupying the spinal canal at the T12 vertebral level (Figs. 1a and b). On MRI, an intradural extramedullary tumor demonstrating low intensity on T2W and T1W was present at the T12 vertebral level, and it was partially enhanced at the margin of the mass on Gd contrast imaging (Figs. 2a-c). The spinal cord was strongly compressed and deviated rightward. Based on these findings, meningioma accompanied 

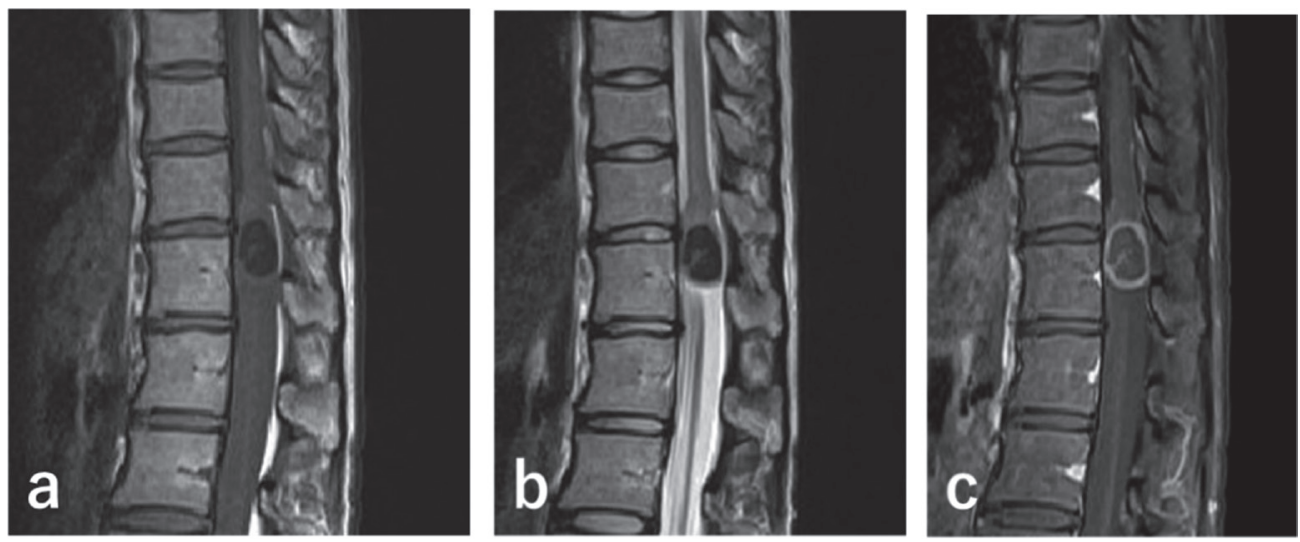

Fig. 2. T1-weighted (a) and T2-weighted (b) sagittal magnetic resonance images showing homogeneous low intensity within the spinal canal at T12 in the patient. T1-weighted gadolinium (c) imaging revealed a round homogenously enhanced tumor, and the signal intensity of the central tumor portion was less enhanced than at the other portions of the mass.

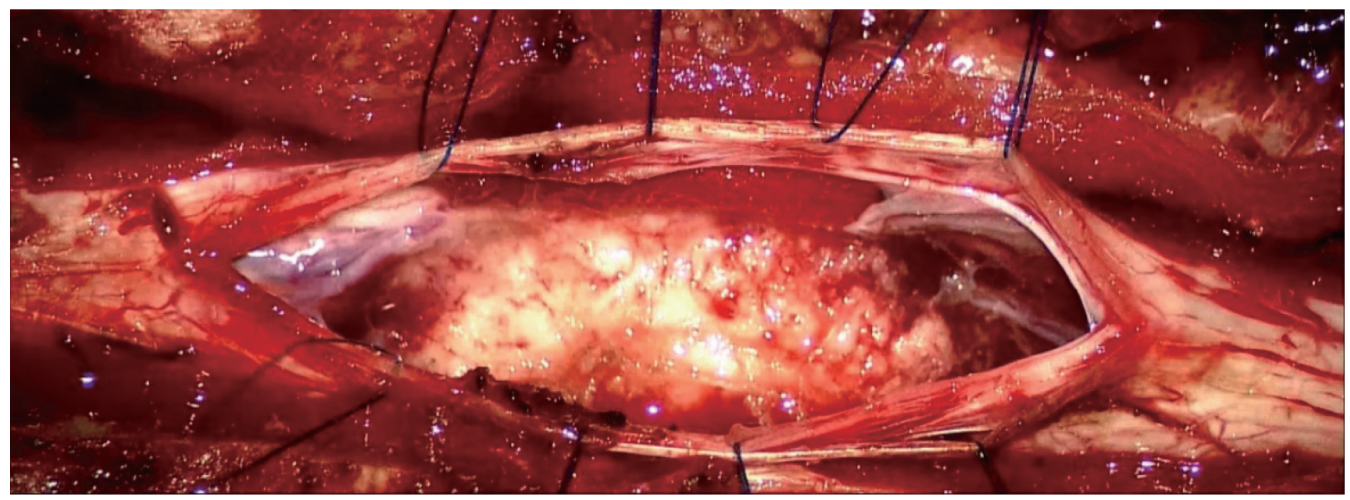

Fig. 3. Intraoperative photograph of the tumor at the T12 level adhered to the arachnoid membrane. The majority of the tumor was very hard.

by ossification was suspected. The patient requested surgery and tumor excision was performed. Surgery was initiated with transcranial motor evoked potentials (MEP) in the prone position under general anesthesia. T10-T12 osteoplastic laminectomy was applied and the dura mater was exposed. The tumor was resected under a microscope. When the dura mater was incised at the midline, a mostly ossified tumor was observed on the left side of the spinal cord; only a small parenchymal component was present (Fig. 3). Excision after debulking and en-bloc resection were considered difficult. The dura mater contacting the tumor was strongly adhered and resection leaving the outer layer was difficult. Thus, the ossified lesion including the dura was resected piece by piece. No change was noted in MEP throughout tumor resection. The defective dural region was repaired with artificial dura mater. Electrical potentials in the left anterior tibial muscle and quadriceps muscle were lost during surgery, but these gradually recovered after resection. Hypersensitivity of the dorsum of the right foot developed after surgery, but muscle strength did not decrease. Postoperative hypersensitivity of the dorsum of the right foot gradually disappeared and the patient was discharged by independent walking 12 days after surgery. As of one year after surgery, only hypoesthesia slightly remained in the right lower limb and the patient was independent in ADL.

Tumor cells comprised groups of cells with an unclear boundary containing an oval nucleus, similar to normal arachnoid cells (meningothelial cell-like tumor cells), cells with an unclear boundary similar to fibroblasts, and cells accompanying collagen tissue (fibrous cell-like cells), being transitional-type meningioma cells. These were arranged in a spiral pattern (whorl formation) and had proliferated. Calcification was noted in the whorl formation and psammoma bodies were present (Figs. $4 \mathrm{a}$ and $\mathrm{b}$ ). The tumor cells were positive for EMA (Fig. 4c). These findings were consistent 



Fig. 4. Photomicrographs of a tumor section. (a) The metaplastic subtype contained highly developed bone formation. Within the tumor, marked heterotopic ossification occurs without psammoma bodies. (Hematoxylin and eosin, bar = $1 \mathrm{~mm}$ ) (b) The meningothelial component had the typical formation of whorls. (Hematoxylin and eosin, bar $=20 \mu \mathrm{m})(\mathbf{c})$ Immunostaining against epithelial membrane antigen was positive in tumor cells $(\mathrm{bar}=20 \mu \mathrm{m})(\mathbf{d})$ Mature osteocytes were present in the bone tissue contained in the tumor tissue, but cells promoting bone formation, such as osteogenic cells and osteoclasts, were absent. (Hematoxylin and eosin, bar $=20 \mu \mathrm{m}$ )

with spinal metaplastic meningioma. Although mature osteocytes were present in the bone tissue within the tumor tissue, basophilic osteoblasts and osteoclasts were not present. Only mature bone tissue and tumor cells were noted in a part of the lesion on HE staining (Fig. $4 d)$, suggesting that rather than the tumor cells having destroyed and invaded mature bone tissue, mature bone tissue had formed in the tumor tissue.

\section{DISCUSSION}

Spinal meningioma has been reported to account for 25 $46 \%$ of all spinal cord tumor cases, ${ }^{1}$ and $87 \%$ of cases at the thoracic vertebral level were in females. ${ }^{5,6}$ Although the most common calcified intradural spinal cord tumor is meningioma, ossified cases are uncommon and account for only $1 \%$ to $5 \%$ of all spinal meningioma. ${ }^{2,3}$ The first case of ossified meningioma was reported by Rogers in $1928 .^{7}$ Ossification is histologically different from calcification. In spinal meningioma, ossification is not always induced by a calcified psammoma body. ${ }^{8}$ In the World Health Organization classification, meningioma accompanied by ossification is histologically classified as a subtype of metaplastic meningioma, and expression of the mesenchymal component is a characteristic. Purely ossified spinal metaplastic meningioma is a very rare pathology and the mechanism of metaplastic ossification remains unclear. The present report is one of few suggesting mature bone tissue formation from tumor cells. Yamane et al. ${ }^{9}$ reviewed 12 cases of ossified spinal meningioma and discussed the ossification mechanism. They stated the following as the most feasible mechanism: Meningioma accompanied by ossification is not secondary to psammomatous features, but it is secondary to metaplasia of arachnoid cells and interstitial cells. Kubota et al. ${ }^{10}$ ultrastructurally investigated the initial mineralization site and mode of calcification in psammoma bodies. They suggested that hydroxyapatite crystals were repeatedly precipitated within the bodies and gradually aggregate within the bodies, resulting in large psammoma bodies. Collagen fibers surrounding the calcified bodies then accumulated deposits of apatite crystals, forming huge psammoma 
bodies. However, Kitagawa et al. ${ }^{11}$ concluded that the ossification of meningioma is secondary to metaplasia of the arachnoid cells rather than psammomatous features. Moreover, Doita et al. ${ }^{8}$ reported that the calcified psammoma bodies may not always lead to bone formation. There are several viewpoints regarding the cause of ossified meningioma, as described above. Chang et al. ${ }^{12}$ reported that in metaplastic meningioma with bone ossification without psammomatous calcification, the extensive calcium deposits formed crystalline structures rather than the characteristic concentric laminations of psammoma bodies on histological examination. Thus, this is the only study to histopathologically demonstrate bone tissue formation from tumor cells. In the present case, mature osteocytes were present in the bone tissue contained in the tumor tissue, but no osteoblasts or osteoclasts were present. Only mature bone tissue and tumor cells were present in the region containing no psammoma bodies, suggesting that the tumor cells transitioned to mature osteocytes, consistent with the previous reports described above.

The authors declare no conflicts of interest.

\section{REFERENCES}

1 Mirimanoff RO, Dosoretz DE, Linggood RM, Ojemann RG, Martuza RL. Meningioma: analysis of recurrence and progression following neurosurgical resection. J Neurosurg. 1985;62:18-24. PMID: 3964853, DOI: 10.3171/ jns.1985.62.1.0018

2 Solero CL, Fornari M, Giombini S, Lasio G, Oliveri G, Cimino C, et al. Spinal meningiomas: review of 174 operated cases. Neurosurgery. 1989;25:153-60. PMID: 2671779, DOI: 10.1227/00006123-198908000-00001
3 Bonstelle CT, Vines FS. Calcification in a cervical intraspinal neurilemmoma. Neuroradiology. 1976;10:231-3. PMID: 934461, DOI: 10.1007/BF00327569

4 Tahir M, Usmani N, Ahmad FU, Salmani S, Sharma MS. Spinal meningioma containing bone: a case report and review of literature. Case Reports. 2009;2009:bcr1120081186. PMID: 21686426, DOI: 10.1136/bcr.11.2008.1186

5 Gezen F, Kahraman S, Çanakci Z, Bedük A. Review of 36 cases of spinal cord meningioma. Spine. 2000;25:727-31. PMID: 10752106, DOI: 10.1097/00007632-200003150-00013

6 Roux FX, Nataf F, Pinaudeau M, Borne G, Devaux B, Meder JF. Intraspinal meningiomas: review of 54 cases with discussion of poor prognosis factors and modern therapeutic management. Surg Neurol. 1996;46:458-64. PMID: 8874546, DOI: 10.1016/S0090-3019(96)00199-1

7 Rogers L. A spinal meningioma containing bone. Br J Surg. 1928;15:675-7. DOI: 10.1002/bjs.1800156015

8 Doita M, Harada T, Nishida K, Marui T, Kurosaka M, Yoshiya S. Recurrent calcified spinal meningioma detected by plain radiograph. Spine. 2001;26:e249-52. PMID: 11389409, DOI: 10.1097/00007632-200106010-00005

9 Yamane K, Tanaka M, Sugimoto Y, Ichimura K, Ozaki T. Spinal metaplastic meningioma with osseous differentiation in the ventral thoracic spinal canal. Acta Med Okayama. 2014;68:313-6. PMID: 25338489

10 Kubota T, Sato K, Yamamoto S, Hirano A. Ultrastructural study of the formation of psammoma bodies in fibroblastic meningioma. J Neurosurg. 1984;60:512-7. PMID: 6699695, DOI: $10.3171 /$ jns.1984.60.3.0512

11 Kitagawa M, Nakamura T, Aida T, Iwasaki Y, Abe H, Nagashima K. [Clinicopathologic analysis of ossification in spinal meningioma]. Noshuyo Byori. 1994;11:115-9. PMID: 8162148

12 Ju CI, Hida K, Yamauchi T, Houkin K. Totally ossified metaplastic spinal meningioma. J Korean Neurosurg Soc. 2013;54:257-60. PMID: 24278660, DOI: 10.3340/ jkns.2013.54.3.257 